\title{
Lysine suppresses myofibrillar protein degradation by regulating the autophagic-lysosomal system through phosphorylation of Akt in $\mathrm{C} 2 \mathrm{C} 12$ cells
}

\author{
Tomonori Sato ${ }^{1}$, Yoshiaki Ito ${ }^{2^{*}}$ and Takashi Nagasawa ${ }^{2}$
}

\begin{abstract}
The prevention of muscle wasting is important for maintaining quality of life, since loss of muscle mass can lead to a bedridden state and decreased resistance to diseases. The prevention of muscle wasting requires an increase in protein synthesis and a decrease in protein degradation in skeletal muscle. We previously showed that lysine (Lys) markedly suppressed myofibrillar protein degradation by inhibiting the autophagic-lysosomal system via the mammalian target of rapamycin (mTOR) and other signal molecules in C2C12 cells. In this study, we investigated the involvement of Akt and adenosine 5'-monophosphate (AMP)-activated protein kinase (AMPK), two regulators of autophagy, on the suppressive effects of Lys on myofibrillar protein degradation in C2C12 cells. Lys induced the phosphorylation of Akt, but the suppressive effects of Lys on myofibrillar protein degradation and autophagy were completely abolished in the presence of Akt1/2 kinase inhibitor (Akti). Lys suppressed the phosphorylation of AMPK, but this effect was also abolished by Akti. On the other hand, AMPK activation by 5-aminoimidazole-4-carboxamide-1- $\beta$-D-ribonucleoside (AICAR) did not affect either Akt activity or the autophagic-lysosomal system in C2C12 cells treated with Lys. These results indicate that regulation of AMPK activity is not essential for the regulation of autophagy by Lys. Taken together, our results show that Lys suppresses myofibrillar protein degradation by the autophagic-lysosomal system through the phosphorylation of Akt in C2C12 cells.
\end{abstract}

Keyword: Lysine; Proteolysis; C2C12; Akt; AMPK

\section{Background}

Skeletal muscle mass reduction is induced when the rate of protein degradation exceeds the rate of protein synthesis in skeletal muscle (Welle 1999). Such changes are caused by several catabolic diseases and by underutilization or disuse of muscle, and lead to muscle wasting (Vary and Lynch 2004; Wolfe 2006; Tisdale 2009). Muscle wasting in turn leads to decreased physical activity and ultimately to a bedridden state. Although appropriate exercise enhances protein synthesis (Mascher et al. 2007; Koopman and van Loon 2009), it is difficult for severely ill patients or the elderly to exercise sufficiently to stimulate protein synthesis. In addition, the cellular and molecular responses to exercise leading to enhanced protein synthesis are decreased in the elderly (Kim et al. 2009). Therefore, a less physically

\footnotetext{
* Correspondence: yito@iwate-u.ac.jp

${ }^{2}$ Department of Biological Chemistry and Food Science, Graduate School of Agriculture, Iwate University, Morioka, Iwate 020-8550, Japan Full list of author information is available at the end of the article
}

straining means7 of preventing muscle wasting is required for the elderly, infirm, and persons with disabilities. The most effective approach for these patients is to improve their nutritional state.

Leucine (Leu), one of the essential amino acids, has been reported to stimulate protein synthesis (Kimball et al. 1999; Lang et al. 2012) and to attenuate protein degradation (Nagasawa et al. 2002; Sugawara et al. 2007). We previously showed that dietary intake of Leu suppresses myofibrillar protein degradation through the autophagic-lysosomal system (Sugawara et al. 2009). Furthermore, we also demonstrated that orally administered Lys suppresses myofibrillar protein degradation in fasted rats (Sato et al. 2013), and that the suppressive effect of Lys on myofibrillar protein degradation is mediated by the autophagic-lysosomal system in $\mathrm{C} 2 \mathrm{C} 12$ myotubes (Sato et al. 2014). In a previous study, we established that the mTOR pathway is involved in the suppressive effect of Lys on the autophagic-lysosomal system 
(Sato et al. 2014). However, the suppressive effect of Lys may be mediated through a signal molecule other than mTOR because the contribution of the mTOR pathway is limited.

We observed the activation of Akt in $\mathrm{C} 2 \mathrm{C} 12$ cells treated with Lys (Sato et al. 2014). Akt is reported to regulate protein degradation through the autophagic-lysosomal system (Arico et al. 2001; Mammucari et al. 2008). AMPK is also known to regulate autophagic-lysosomal activity (Sanchez et al. 2012). In addition, it has been reported that Akt activity negatively regulates phosphorylation of AMPK (Kovacic et al. 2003). Although Akt phosphorylation is induced by Lys, the involvement of Akt phosphorylation on the regulation of myofibrillar protein degradation and on AMPK activity remains unclear.

In this study, we investigated whether Lys suppresses myofibrillar protein degradation through regulation of the Akt and/or AMPK pathway in $\mathrm{C} 2 \mathrm{C} 12$ cells.

\section{Materials and methods Materials}

Fetal bovine serum (FBS) and horse serum (HS) were purchased from BioWest (Nuaillé, France) and Invitrogen (Carlsbad, CA, USA), respectively. Dulbecco's modified Eagle's medium (DMEM, low glucose), minimum essential medium (MEM) Vitamin mix, Akti and dimethyl sulfoxide (DMSO) were obtained from Sigma (St. Louis, MO, USA). HEPES was obtained from Dojindo Molecular Technologies, Inc. (Kumamoto, Japan). LC3B antibody, AMPK $\alpha$ rabbit mAb, phospho-AMPK $\alpha$ (Thr172) rabbit mAb and AICAR were obtained from Cell Signaling Technology, Inc. (Danvers, MA, USA). 4E-BP1 (R-113), Akt1 (B-1) and p-Akt 1/2/3 (Ser 473) were obtained from Santa Cruz Biotechnology, Inc. (Santa Cruz, CA, USA). HRP-conjugated goat anti-rabbit IgG and HRP-conjugated goat anti-mouse IgG were obtained from Kirkegaard \& Perry Laboratories, Inc. (Gaithersburg, MD, USA). Other chemicals were obtained from Wako Pure Chemical Industries Ltd. (Osaka, Japan).

\section{Cell culture}

C2C12 myoblasts $\left(5.0 \times 10^{4}\right.$ cells $\left./ \mathrm{cm}^{2}\right)$ were seeded into DMEM containing antibiotics (100 units $/ \mathrm{mL}$ penicillin and $100 \mu \mathrm{g} / \mathrm{mL}$ streptomycin), 10\% (v/v) FBS and $44 \mathrm{mM}$ sodium bicarbonate, and then cultured for 2 days. Fusion and differentiation of myoblasts into myotubes were induced by replacing the medium containing $10 \%$ FBS with medium containing $2 \%(\mathrm{v} / \mathrm{v})$ HS. Fusion and differentiation conditions were maintained for 6 days, during which the medium was changed daily.

\section{Measurement of myofibrillar protein degradation}

Since 3-methylhistidine (MeHis) is primarily in myosin and actin, two myofibrillar proteins and is not reused for protein synthesis (Young and Munro 1978), the rate of
MeHis release from muscle cells directly reflects the rate of myofibrillar protein degradation (Sato et al. 2014). We measured the rates of $\mathrm{MeHis}$ release from $\mathrm{C} 2 \mathrm{C} 12$ as described previously (Sato et al. 2014). In brief, myotube cells were rinsed twice with phosphate buffered saline (PBS) after 6 days of differentiation. Then, the cells were cultured for $4 \mathrm{~h}$ in serum and amino acid (AA)-deficient medium $\left((\mathrm{C})\right.$ : $\left[1 \mathrm{mg}\right.$ ferric nitrate $\cdot 9 \mathrm{H}_{2} \mathrm{O} / \mathrm{L}, 0.4 \mathrm{~g} \mathrm{KCl} / \mathrm{L}$, $6.4 \mathrm{~g} \mathrm{NaCl} / \mathrm{L}, 0.142 \mathrm{~g} \mathrm{NaH}_{2} \mathrm{PO}_{4} \cdot 2 \mathrm{H}_{2} \mathrm{O} / \mathrm{L}, 1.0 \mathrm{~g}$ glucose/L, $0.265 \mathrm{~g} \mathrm{CaCl}_{2} \cdot 2 \mathrm{H}_{2} \mathrm{O} / \mathrm{L}, 0.2 \mathrm{~g} \mathrm{MgSO}_{4} \cdot 7 \mathrm{H}_{2} \mathrm{O} / \mathrm{L}, 0.1 \%$ $(\mathrm{w} / \mathrm{v})$ bovine serum albumin (BSA), 1\% (v/v) MEM Vitamin mix, $20 \mathrm{mM}$ HEPES and antibiotics]), serum and AA-deficient medium containing $10 \mu \mathrm{M}$ Akti (Ai), serum and AA-deficient medium containing $10 \mathrm{mM}$ Lys $(\mathrm{K})$, serum and AA-deficient medium containing $10 \mathrm{mM}$ Lys and $10 \mu \mathrm{M}$ Akti (AiK), serum and AAdeficient medium containing $1 \mathrm{mM}$ AICAR (Ac), or serum and AA-deficient medium containing $10 \mathrm{mM}$ Lys and $1 \mathrm{mM}$ AICAR (AcK). The rate of myofibrillar protein degradation was measured during the $4 \mathrm{~h}$ culture period.

\section{Western blot analysis}

After 6 days of differentiation, the myotube cells were rinsed twice with PBS and incubated with DMEM containing $0.1 \%(\mathrm{w} / \mathrm{v})$ BSA for $6 \mathrm{~h}$. Then, the medium was replaced with new DMEM containing 0.1\% (w/v) BSA, DMEM containing $0.1 \%(\mathrm{w} / \mathrm{v})$ BSA and $10 \mathrm{mM}$ Leu, DMEM containing $0.1 \%(\mathrm{w} / \mathrm{v})$ BSA and $10 \mathrm{mM}$ Lys, or DMEM containing $0.1 \%(\mathrm{w} / \mathrm{v}) \mathrm{BSA}$ and $10 \mathrm{mM}$ Gly. Akti $(10 \mu \mathrm{M})$ or AICAR $(1 \mathrm{mM})$ dissolved in DMSO (final concentration of $0.05 \%(\mathrm{v} / \mathrm{v})$ ) or DMSO alone (final concentration of $0.05 \%(\mathrm{v} / \mathrm{v})$ ) was added to the medium 30 min before amino acid stimulation. Myotubes in DMEM containing $0.1 \%(\mathrm{w} / \mathrm{v})$ BSA with $10 \mathrm{mM}$ Leu (L), $10 \mathrm{mM}$ Lys (K), $10 \mathrm{mM}$ Gly (G), or without amino acids supplementation (C) were cultured for $30 \mathrm{~min}$, treated with lysis buffer solution (1\% (v/v) Triton$\mathrm{X}, 5 \%(\mathrm{w} / \mathrm{v})$ deoxycholic acid, $0.1 \%(\mathrm{v} / \mathrm{w})$ sodium dodecyl sulfate (SDS), $20 \mathrm{mM}$ Tris- $\mathrm{HCl}$ (pH 7.4), $150 \mathrm{mM}$ sodium chloride, $0.5 \mathrm{mM}$ sodium orthovanadate (V) and $5 \mathrm{mM}$ EDTA), then collected. Collected myotubes were centrifuged at $4^{\circ} \mathrm{C}, 17,900 \times g$ for $10 \mathrm{~min}$, then the supernatant was subjected to SDS-PAGE. Equal amounts of protein from each of the samples were separated on $10 \%$ SDS-PAGE gels, then transferred to a PVDF membrane (Millipore Corporation, Billerica, MA, USA). The membrane was blocked for $1 \mathrm{~h}$ with $5 \%$ skim milk in Tris buffered saline (TBS) containing $0.1 \%$ Tween 20 (TBS-T) at room temperature. The membrane was incubated overnight at $4^{\circ} \mathrm{C}$ with primary antibodies, then the membrane was incubated with HRP-conjugated goat anti-rabbit IgG or HRP-conjugated goat anti-mouse IgG in TBS-T. The secondary antibody was detected using an ECL western 
blot detection kit (GE Healthcare, Tokyo, Japan). The bands were scanned using a luminescent image analyzer (ImageQuant LAS 4000, GE Healthcare) and the relative intensity of each band was estimated using NIH Image.

\section{Gene expression of ubiquitin-ligase}

The myotube cells were rinsed twice with PBS after 6 days of differentiation, then cultured in serum and AA-deficient medium for $1 \mathrm{~h}$. Then, the cells were incubated in serum and AA-deficient medium $(\mathrm{C})$ containing either $1 \mathrm{mM}$ AICAR (Ac), $10 \mathrm{mM}$ Lys (K), or $10 \mathrm{mM}$ Lys and $1 \mathrm{mM}$ AICAR (AcK), for $30 \mathrm{~min}$ or $4 \mathrm{~h}$. Subsequently, the mRNA expressions of E3 ubiquitin ligases, muscle ring-finger protein 1 (MuRF1) and atrogin-1 were measured. Total RNA was extracted from the treated cells at the indicated time. Ten micrograms of total RNA from each $\mathrm{C} 2 \mathrm{C} 12$ culture were separated on a $1.2 \%$ agarose-formaldehyde gel and transferred to a positively-charged nylon membrane (Pall Corporation, Port Washington, NY, USA). After UV cross-linking, membranes were hybridized with a digoxigenin-labeled cDNA probe specific to MuRF1, atrogin-1 or GAPDH for 12 to $16 \mathrm{~h}$ at $50^{\circ} \mathrm{C}$ in hybridization solution (5× SSC, $50 \%$ formamide, $50 \mathrm{mM}$ sodium phosphate buffer, $\mathrm{pH} 7.0$, 7\% SDS, 2\% blocking reagent (Roche Diagnostics, Mannheim, Germany), and $0.1 \%$ N-lauroylsarcosine). Membranes were washed twice with $2 \times$ SSC-0.1\% SDS for $15 \mathrm{~min}$ at room temperature and twice with $0.1 \times$ SSC$0.1 \% \mathrm{SDS}$ for $15 \mathrm{~min}$ at $68^{\circ} \mathrm{C}$. Specific hybridization was detected with an anti-digoxigenin antibody conjugated with alkaline phosphatase, and blots were developed with CDP-star reagent (Tropix, Bedford, MA, USA). The bands were scanned using a luminescence image analyzer (ImageQuant LAS 4000) and the relative intensity of each band was estimated using NIH Image.

\section{Statistical analysis}

Data are expressed as mean with SE. Data analysis was performed using GraphPad Instat Software version 3.0a (2001, GraphPad Software, Inc., San Diego, CA, USA). Data were analyzed by analysis of variance (ANOVA) and Tukey's post-test in multigroup comparisons to determine whether there were significant differences $(\mathrm{p}<0.05)$ among the groups.

\section{Results}

Activation of the Akt pathway by Lys suppresses myofibrillar protein degradation through regulation of the autophagic-lysosomal system

The phosphorylation levels of Akt Ser473 and Thr308 were significantly increased upon stimulation with $10 \mathrm{mM}$ Leu (L) or $10 \mathrm{mM}$ Lys (K), and these upregulations of Akt phosphorylation were abolished by Akti (Figure 1).
To investigate whether the suppressive effect of Lys on myofibrillar protein degradation depends on the activation of Akt, we measured the rate of myofibrillar protein degradation in $\mathrm{C} 2 \mathrm{C} 12$ myotubes treated with $10 \mathrm{mM}$ Lys and $10 \mu \mathrm{M}$ Akti. Lys did not suppress myofibrillar protein degradation when Akt activity was inhibited by Akti (AiK), whereas treatment with Lys alone (K) markedly suppressed myofibrillar protein degradation (Figure 2).

Light chain 3 (LC3) is a mammalian homolog of Apg8p, a protein that is essential for the autophagiclysosomal system in yeast. LC3 has two forms: LC3-I and LC3-II. The ratio of LC3-II to total LC3 (LC3-I + LC3-II) was used as a marker of autophagy (Naito et al. 2013). We evaluated the involvement of Akt activation induced by Lys in the regulation of autophagic-lysosomal activity by the ratio of LC3-II to total LC3. The ratio of LC3-II to total LC3 was reduced in C2C12 cells treated with Lys (K), however, this reduction was abolished by treatment with Akt inhibitor (AiK). Similar results were observed when the cells were treated with Leu (Figure 3). These results suggest that the suppression of the autophagic-lysosomal system by Lys is dependent on the activation of Akt.

\section{Lys regulates the mTOR and AMPK pathways through activation of Akt}

Next, we investigated the effect of Akt activation by Leu and Lys on the mTOR and AMPK pathways. One downstream target of mTOR, eIF4E-binding protein 1 (4E-BP1), was significantly phosphorylated by treatment with Leu (L) or Lys (K), and the up-regulation of 4E-BP1 phosphorylation was abolished by Akti (AiK) (Figure 4a). The level of AMPK phosphorylation was suppressed by $40 \%$ when the medium was supplemented with Leu or Lys, and the suppressive effect of Lys on AMPK phosphorylation was abolished by Akti (Figure 4b). These results indicated that Lys regulates the mTOR and AMPK pathways through Akt activation.

\section{Akt plays a dominant role in the regulation of autophagic-lysosomal activity compared to the AMPK pathway}

We addressed the physiological role of the regulation of AMPK activity by Akt. To do this, we examined three effects of AICAR: on the phosphorylation of Akt, on autophagic-lysosomal activity, and on MeHis release upon Lys treatment. It was previously reported that the AMPK pathway induces myofibrillar protein degradation (Nakashima and Yakabe 2007). We found that AICAR treatment induced AMPK activation, and that this activation was not suppressed by Leu or Lys treatment (Figure 5a). Activation of Akt by Leu or Lys was also not affected by AICAR treatment (Figure $5 \mathrm{~b}$ ). The ratio of 

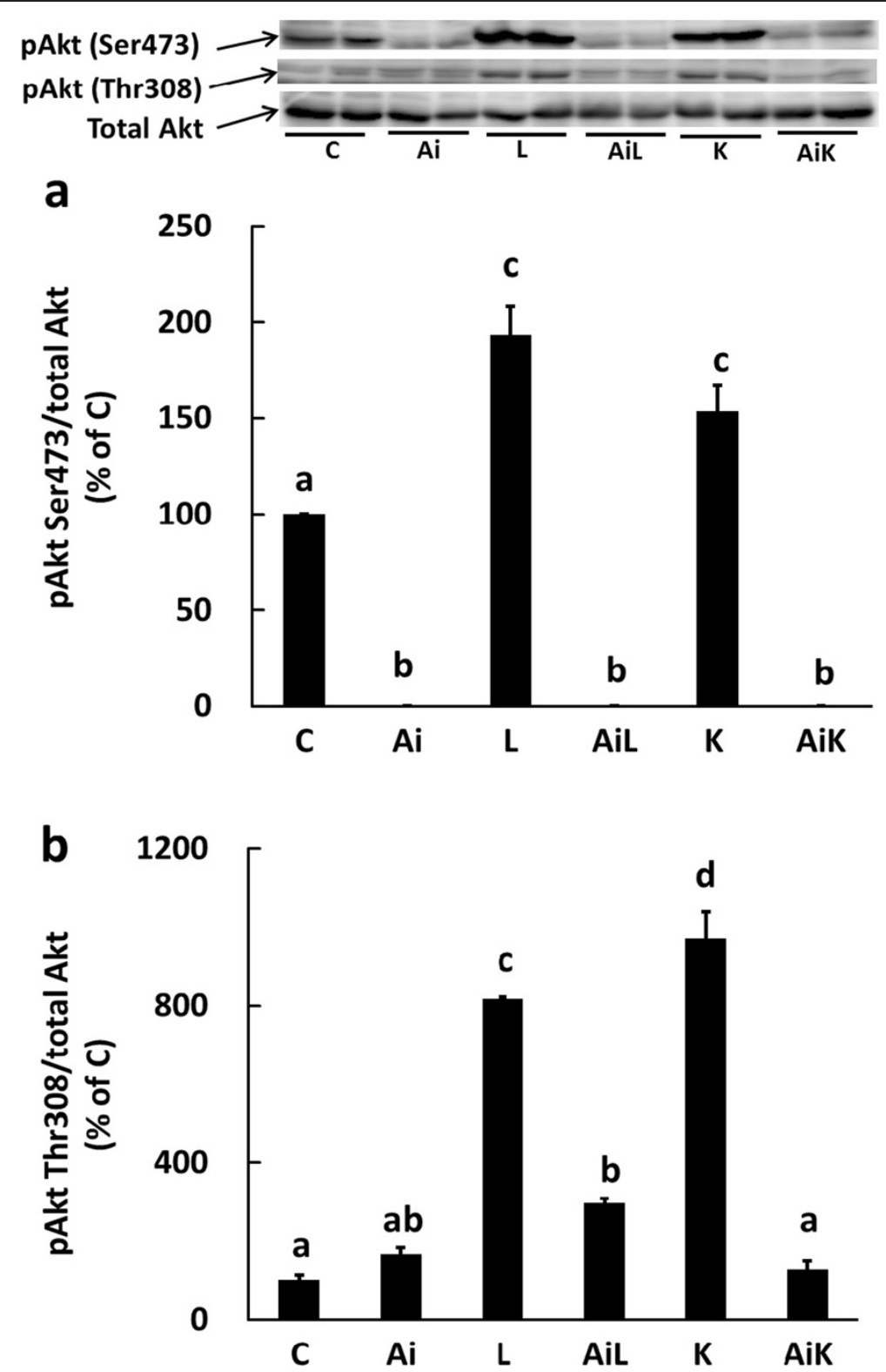

Figure 1 Lys induces phosphorylation of Akt in C2C12 myotubes. C2C12 myotubes were treated for 30 min with DMSO (C), $10 \mu M$ Akti (Ai), $10 \mathrm{mM}$ Leu (L), $10 \mathrm{mM}$ Leu and $10 \mu \mathrm{M}$ Akti (AiL), $10 \mathrm{mM}$ Lys (K), or $10 \mathrm{mM}$ Lys and $10 \mu \mathrm{M}$ Akti (AiK). The phosphorylation level of Akt Ser473 (a) and Akt Thr308 (b) in the lysates was analyzed by Western blotting. Results are expressed as the level relative to the expression level of the control group. Representative immunoblots are shown. Values are means with SE $(n=3-4)$. Different letters indicate significant differences among the groups $(p<0.05)$

LC3-II to total LC3 was unaffected by AICAR treatment, but the ratio decreased when Leu or Lys was added to the medium, even in the presence of AICAR (Figure $5 \mathrm{~d}$ ). These results suggested that Akt, rather than AMPK pathway, might dominantly regulate autophagiclysosomal activity.

The mTOR pathway is suppressed by the AMPK pathway (Bolster et al. 2002), and the mTOR pathway is involved in the suppressive effect of Lys on autophagic-lysosomal activity (Sato et al. 2014). Consequently, we investigated whether mTOR activity is affected by AMPK activation. Activation of the AMPK pathway by AICAR decreased the basal phosphorylation level of 4E-BP1 and reduced the phosphorylation levels of 4E-BP1 induced by Leu or Lys (Figure 5c). These results suggest that mTOR activity may not be critical for the regulation of autophagic-lysosomal activity by Lys.

MeHis release is an index of myofibrillar protein degradation. MeHis release increased in the presence of AICAR and Lys compared to in the presence of Lys 


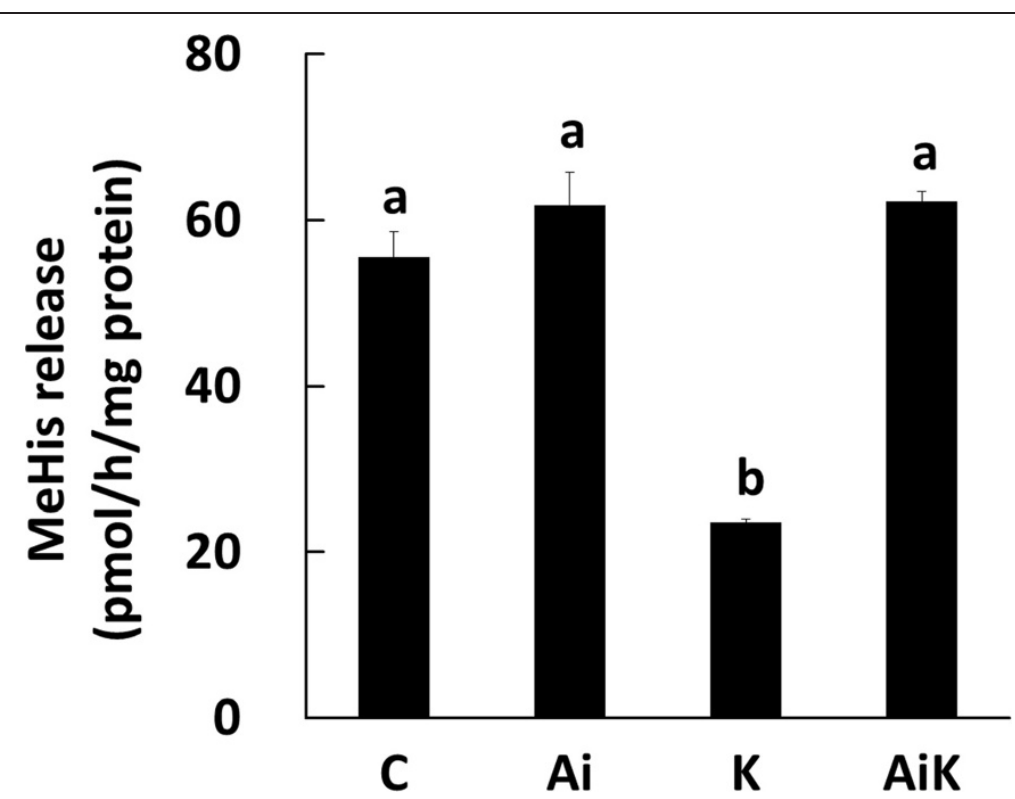

Figure 2 Lys suppresses myofibrillar protein degradation through activation of Akt in C2C12 myotubes. Cells were incubated in medium containing DMSO (C), $10 \mu \mathrm{M}$ Akti (Ai), $10 \mathrm{mM}$ Lys (K), or $10 \mathrm{mM}$ Lys with $10 \mu \mathrm{M}$ Akti (AiK) for $4 \mathrm{~h}$. The amount of MeHis released from the cells was measured by HPLC. Values are means with SE $(n=3)$. Different letters indicate significant differences among the groups $(p<0.05)$.
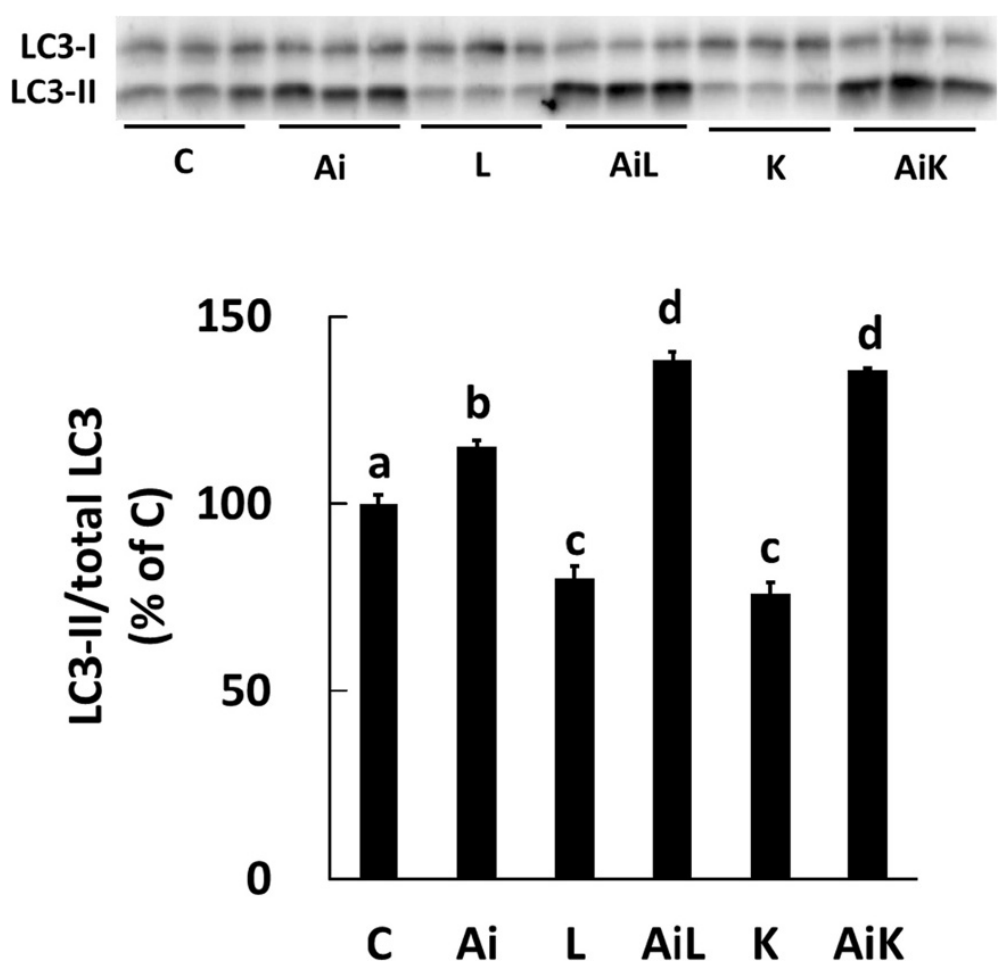

Figure $\mathbf{3}$ Lys suppresses the autophagic-lysosomal system through activation of Akt in C2C12 myotubes. Cells were treated for 30 min with DMSO (C), $10 \mu \mathrm{M}$ Akti (Ai), $10 \mathrm{mM}$ Leu (L), $10 \mathrm{mM}$ Leu and $10 \mu \mathrm{M}$ Akti (AiL), $10 \mathrm{mM}$ Lys (K), or $10 \mathrm{mM}$ Lys and $10 \mu \mathrm{M}$ Akti (AiK). The ratio of LC3-II to total LC3 (LC3-I + LC3-II) in the lysates was determined by Western blotting. Results are expressed as the level relative to the control group. Values are means with SE $(n=3-4)$. Different letters indicate significant differences among the groups $(p<0.05)$. 


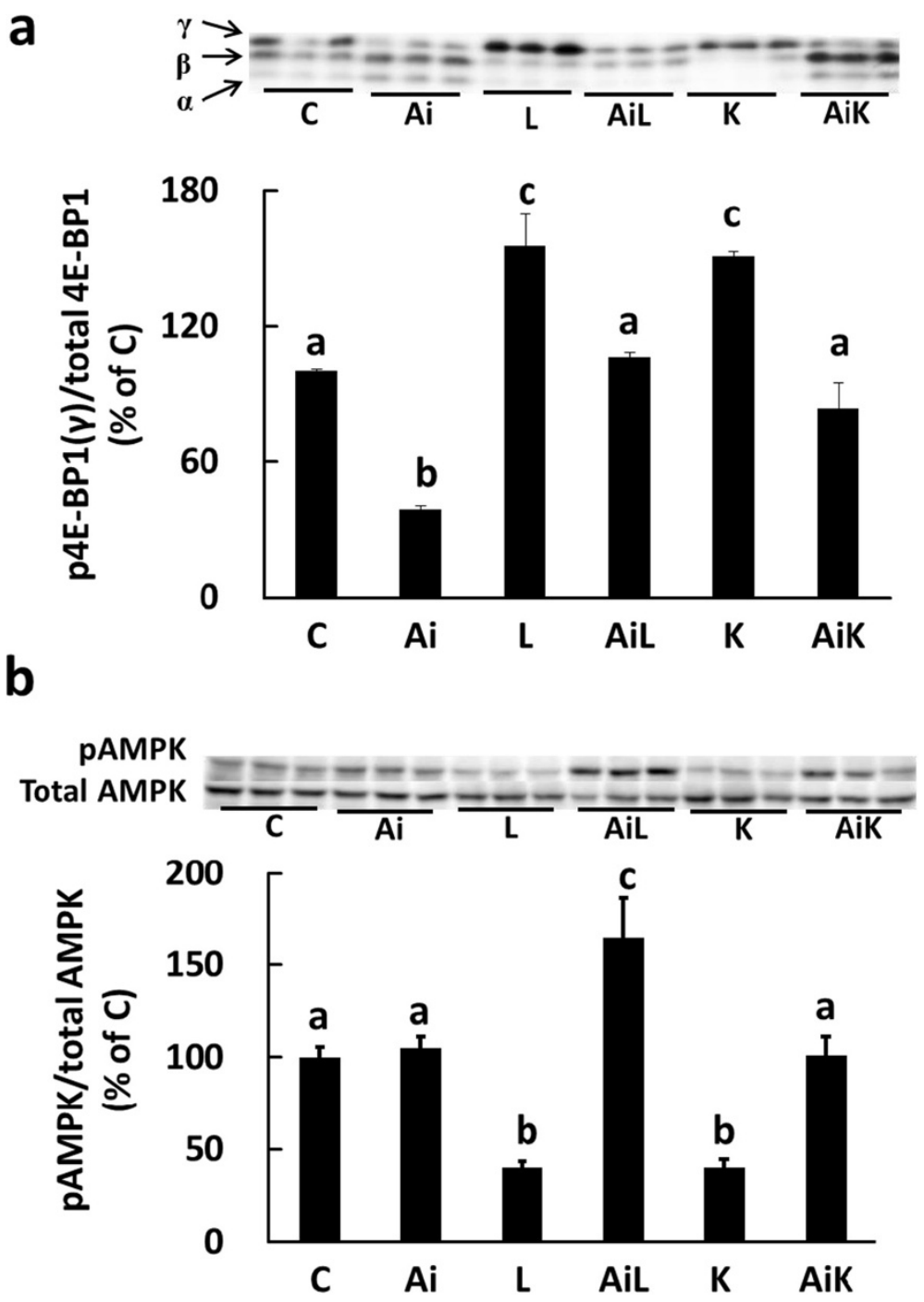

Figure 4 Lys regulates the mTOR and AMPK pathways by activating Akt in C2C12 myotubes. Cells were treated for 30 min with DMSO (C), $10 \mu \mathrm{M}$ Akti (Ai), $10 \mathrm{mM}$ Leu (L), $10 \mathrm{mM}$ Leu and $10 \mu \mathrm{M}$ Akti (AiL), $10 \mathrm{mM}$ Lys (K), or $10 \mathrm{mM}$ Lys and $10 \mu \mathrm{M}$ Akti (AiK). The phosphorylation level of 4E-BP1 (a) and AMPK (b) in the lysates was analyzed by Western blotting. The level of 4E-BP1 phosphorylation was estimated from the ratio of the $\gamma$-form to that of total $4 \mathrm{E}-\mathrm{BP}$. Results are expressed as the level relative to the level in the control group. Representative immunoblots are shown. Values are means with SE $(n=3-4)$. Different letters indicate significant differences among the groups $(p<0.05)$.

alone (Figure 6). On the other hand, autophagiclysosomal activity (the ratio of LC3-II to total LC3) was sufficiently suppressed by Lys in the presence of AICAR (Figure 5d). AICAR treatment was reported to induce the expression of the E3 ubiquitin ligase genes, MuRF1 and atrogin-1, in C2C12 myotubes (Nakashima and Yakabe 2007); therefore, we examined whether AICAR treatment stimulates the ubiquitin-proteasomal system. The expression of MuRF1 and atrogin-1 mRNA significantly increased in $\mathrm{C} 2 \mathrm{C} 12$ cells treated with Lys and AICAR (AcK), whereas treatment with either AICAR or Lys alone (Ac, K) did not affect MuRF1 and atrogin-1 mRNA levels (Figure 7). Therefore, the increase in MeHis release may reflect activation of the ubiquitin-proteasomal system in cells treated with Lys and AICAR.

Glycine slightly induces Akt activation and does not regulate the phosphorylation of AMPK and the autophagic-lysosomal system

We previously showed that Gly does not suppress myofibrillar protein degradation in $\mathrm{C} 2 \mathrm{C} 12$ myotubes (Sato et al. 2014). However, it is still unknown whether Gly regulates the activities of autophagy and signal molecules which regulate autophagy. We therefore investigated the effect of Gly on the phosphorylation levels of Akt and AMPK, and on the activity of the autophagic- 

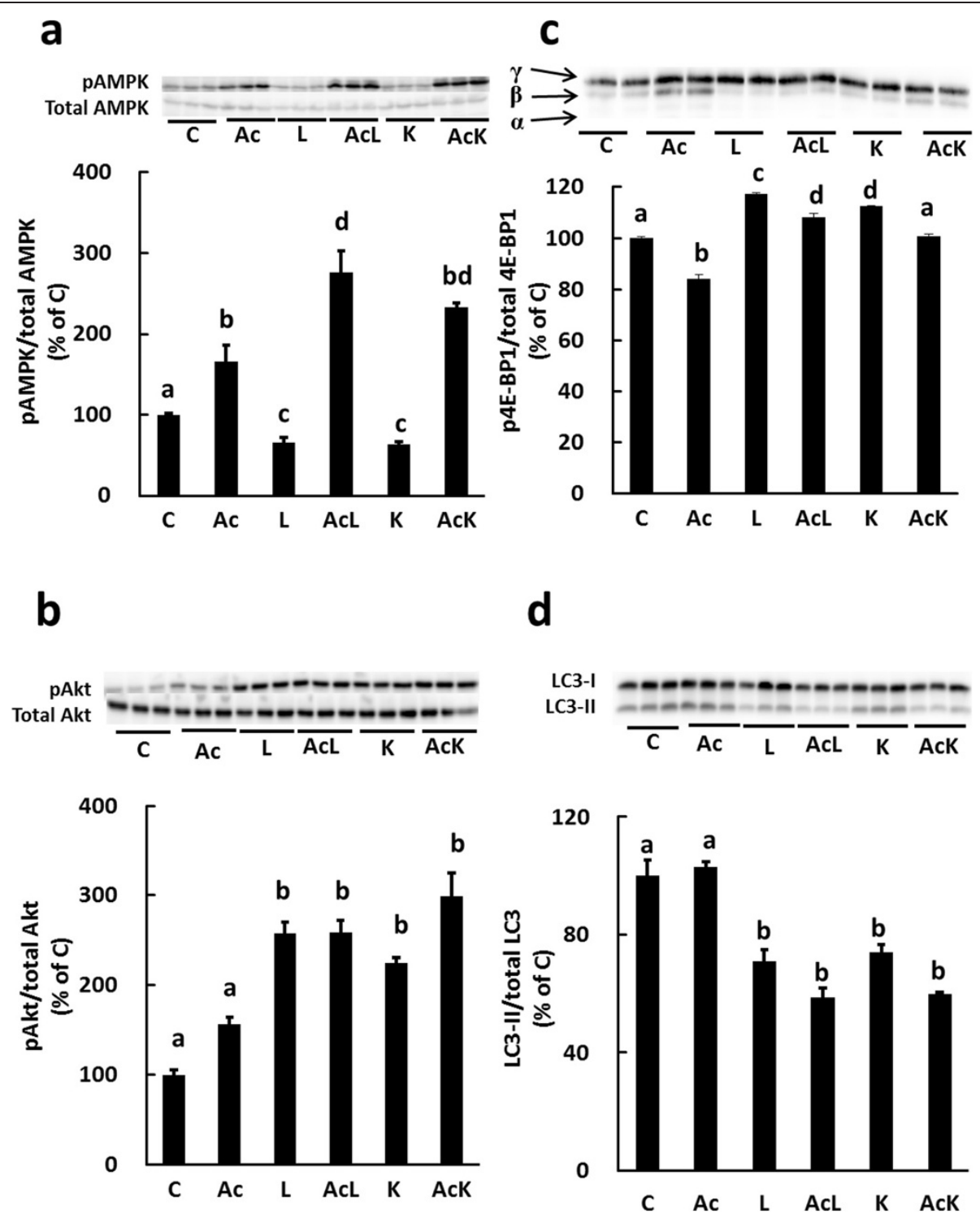

Figure 5 Effect of Lys and/or AICAR treatment on activation of the AMPK, Akt and mTOR pathways, and on the autophagic-lysosomal system in C2C12 myotubes. Cells were treated with DMSO (C), $1 \mathrm{mM}$ AICAR (Ac), $10 \mathrm{mM}$ Leu (L), $10 \mathrm{mM}$ Leu and $1 \mathrm{mM}$ AICAR (AcL), $10 \mathrm{mM}$ Lys (K), or $10 \mathrm{mM}$ Lys and $1 \mathrm{mM}$ AICAR (AcK) for $30 \mathrm{~min}$. The lysates were analyzed for phosphorylation levels of AMPK (a), Akt (b) and 4E-BP1 (c), and the ratio of LC3-II to total LC3 (LC3-I + LC3-II) (d), by Western blotting. Results are expressed as the level relative to the control group. Values are means with SE $(n=3-4)$. Different letters indicate significant differences among the groups $(p<0.05)$.

lysosomal system in C2C12 myotubes. The phosphorylation level of Akt Ser473 slightly increased upon Gly treatment, but the level was much lower than that obtained following Lys stimulation. In addition, the phosphorylation level of Akt Thr308 did not increase upon Gly treatment (Figure 8a, b). Furthermore, Gly did not affect the phosphorylation of AMPK (Figure 8c), and the ratio of LC3-II to total LC3 was not changed by Gly treatment (Figure $8 \mathrm{~d}$ ). These results demonstrate that regulation of the Akt and AMPK pathways, and of the autophagic-lysosomal system, is specific to Lys, and that the effect of Lys is not merely due to it being a supplemental nitrogen source.

\section{Discussion}

Leu is known to activate mTOR signaling, which stimulates muscle protein synthesis and suppresses protein degradation (Kimball et al. 1999; Kim et al. 2009). We previously showed that Lys suppresses myofibrillar protein degradation through mTOR signaling and another signaling pathway, and we observed significant upregulation of Akt phosphorylation by Lys (Sato et al. 2014). 


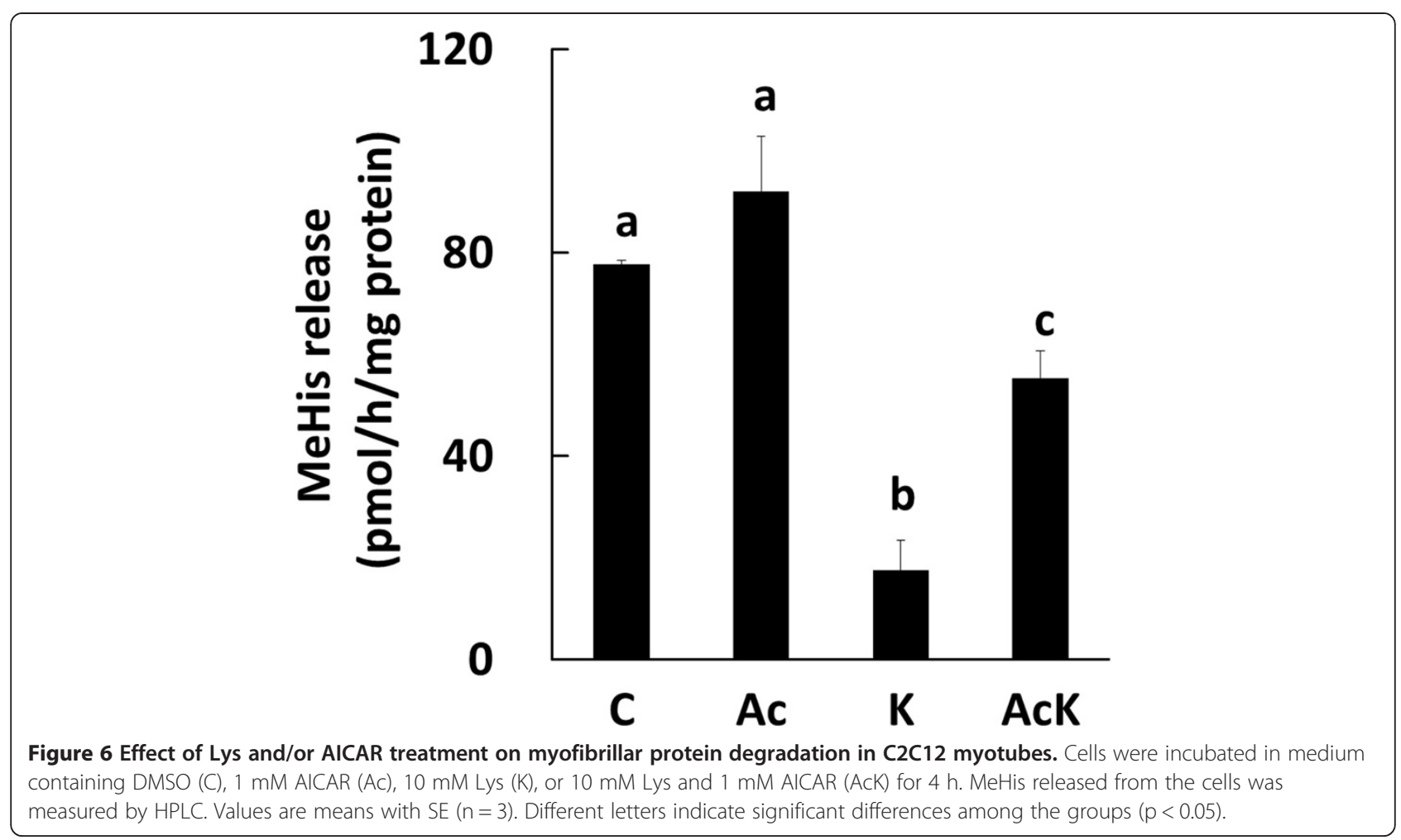

Akt has been reported to regulate the autophagic-lysosomal system (Arico et al. 2001; Mammucari et al. 2008). Therefore, we here investigated whether Lys suppresses myofibrillar protein degradation by regulating the phosphorylation of Akt in C2C12 myotubes.

The phosphorylation level of Akt Ser473 significantly increased with Lys treatment (Figure 1a), as shown previously (Sato et al. 2014), and the phosphorylation level of Akt Thr308 also significantly increased in the presence of Lys (Figure 1b). The suppressive effect of Lys on myofibrillar protein degradation was completely abolished when Akt phosphorylation was inhibited by Akti (Figures 1 and 2). These results clearly demonstrate that Lys suppresses myofibrillar protein degradation by activating Akt. Similarly, the autophagic-lysosomal system in $\mathrm{C} 2 \mathrm{C} 12$ cells treated with Akti was not suppressed by Lys, since the ratio of LC3-II to total LC3 did not decrease (Figure 3). This result indicates that activation of Akt is essential for the suppression of the autophagiclysosomal system by Lys.

Several studies have shown that Akt is activated by the addition of amino acids. Tato et al. (2011) showed that Akt is phosphorylated following the addition of a mixture of amino acids, and Leu has been reported to activate Akt phosphorylation (Lee et al. 2008; Coëffier et al. 2011; Zeanandin et al. 2012). These studies support our finding that Lys is another stimulating amino acid that can activate the Akt pathway (Figure 1). However, the effect on Akt activation seems to be specific to certain amino acids, as Gly only slightly stimulated Akt phosphorylation and did not suppress autophagic-lysosomal activity (Figure 8). Therefore, Lys may be an important regulatory amino acid in muscle protein metabolism.

It has been reported that Akt suppresses autophagy via mTOR signaling (Janku et al. 2011; Jamart et al. 2012; Seldin et al. 2013). In this study, we found that activation of Akt by Lys regulates mTOR signaling (Figure 4a). However, we previously showed that mTOR signaling plays a limited role in the suppression of myofibrillar protein degradation by Lys (Sato et al. 2014), and it has been shown that Leu also regulates the autophagiclysosomal system in an mTOR-independent manner (Mordier et al. 2000). Furthermore, Zhao et al. (2007) have shown that Akt, rather than mTOR, is a master regulator of autophagy in myotubes. Therefore, it is likely that the activation of Akt plays a primary role in the regulation of autophagic-lysosomal activity by Lys. In this study, we observed that Leu also regulates some signals in similar manner to Lys. From these results, the regulatory mechanism on autophagy by Lys may be similar to that by Leu. However, the stimulatory effect of Leu on mTOR signaling was significantly higher than that of Lys in this study (Figure 5c) and previous study (Sato et al. 2014). Therefore, the regulation of mTOR signaling or protein synthesis by Lys may be weaker than that by Leu. Further study is needed to clarify that similarity and difference between Leu and Lys signals on regulation of protein metabolism. 


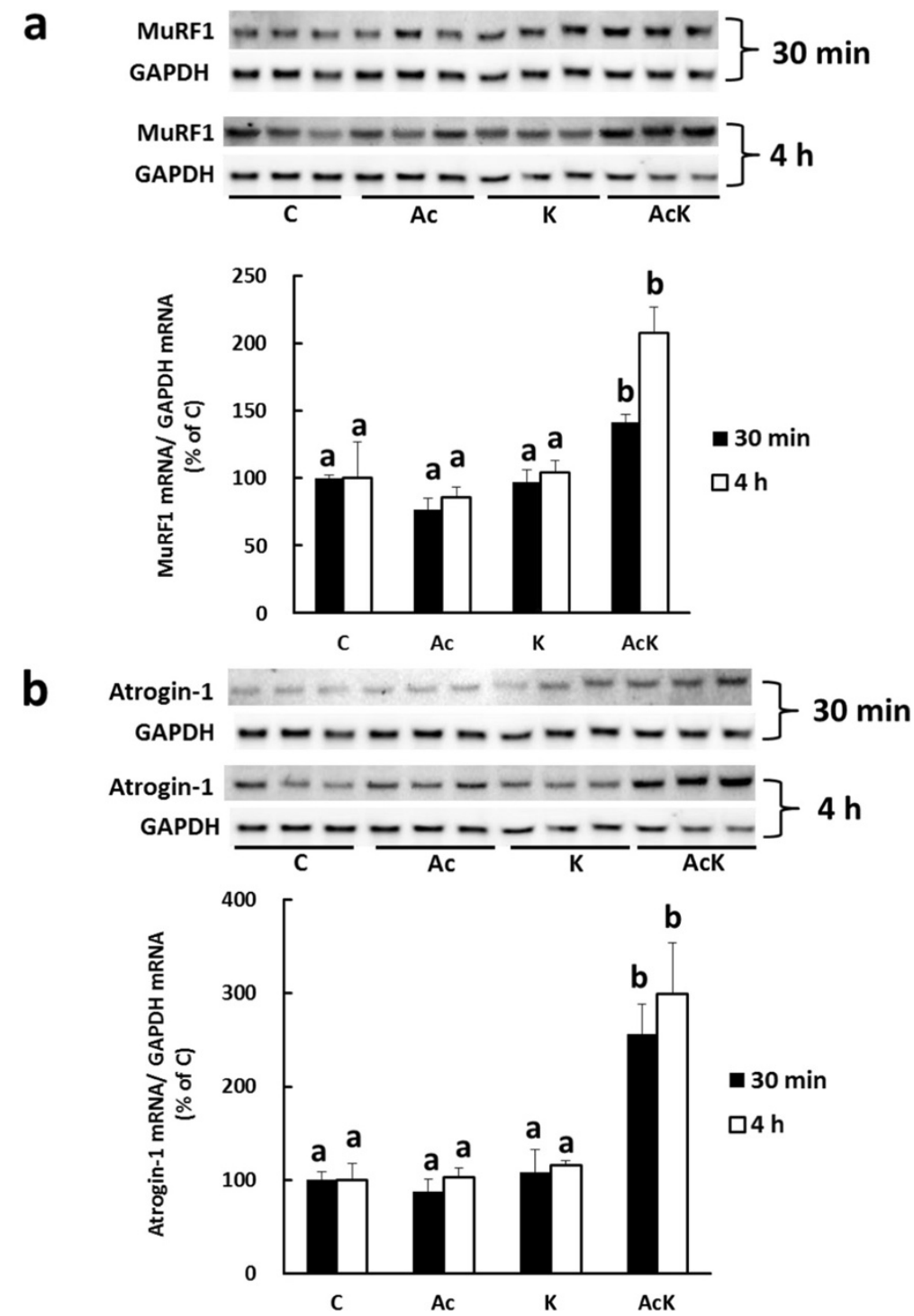

Figure 7 Effect of Lys and/or AICAR on gene expression of MuRF1 and atrogin-1 mRNA in C2C12 myotubes. Cells were treated with DMSO (C), 1 mM AICAR (Ac), $10 \mathrm{mM}$ Lys (K), or $10 \mathrm{mM}$ Lys and $1 \mathrm{mM}$ AICAR (AcK) for $30 \mathrm{~min}$ (filled bar) or $4 \mathrm{~h}$ (open bar). The expression of MuRF1 (a) and atrogin-1 (b) mRNA was analyzed as described in "Materials and Methods". Results are shown as expression relative to the control group. Values are means with SE $(n=3)$. Different letters indicate significant differences among the groups at the same time point $(p<0.05)$.

AMPK is also a regulator of autophagy (Sanchez et al. 2012). Leu has been reported to regulate protein turnover by suppressing AMPK (Du et al. 2007; Wilson et al. 2011). In this study, we showed that Lys suppresses AMPK phosphorylation, and that the suppressive effect of Lys on AMPK phosphorylation was completely abolished when Akt activity was inhibited (Figure 4b). Leu similarly regulated AMPK phosphorylation by Akt in C2C12 cells. AMPK is a downstream target of Akt (Kovacic et al. 2003; Horman et al. 2006; Bertrand et al. 2008; Suzuki et al. 2013). Therefore, the current results indicate that both Lys and Leu suppress AMPK phosphorylation through Akt activation in $\mathrm{C} 2 \mathrm{C} 12$ cells.
C2C12 myotubes were treated with AICAR for $4 \mathrm{~h}$ to examine the involvement of AMPK activity in the degradation of myofibrillar protein. Treatment with AICAR alone had essentially no effect on MeHis release from cells (Figure 6). In contrast, Nakashima and Yakabe (2007) showed that activation of AMPK by AICAR stimulates myofibrillar protein degradation in $\mathrm{C} 2 \mathrm{C} 12$ cells. This discrepancy between the two sets of results may be due to the different treatment times ( $24 \mathrm{~h}$ in the study by Nakashima and Yakabe, and $4 \mathrm{~h}$ in this study); alternatively, AMPK activity might have been sufficiently high in the current study to promote the degradation of myofibrillar protein even under basal conditions without 
a
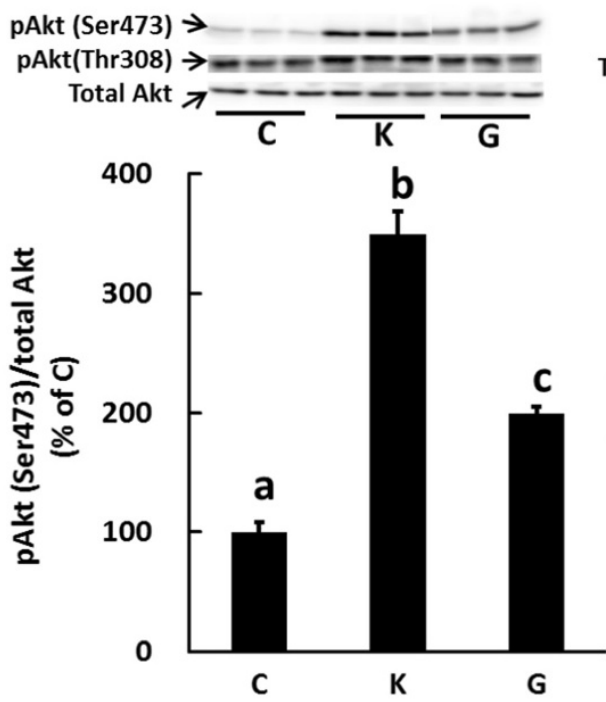

b

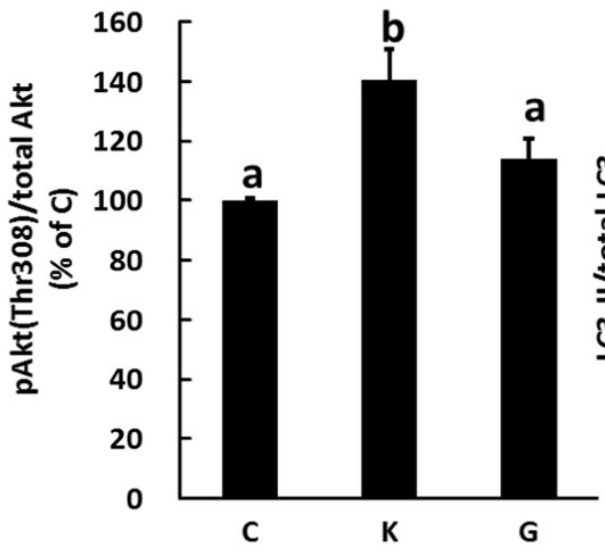

C
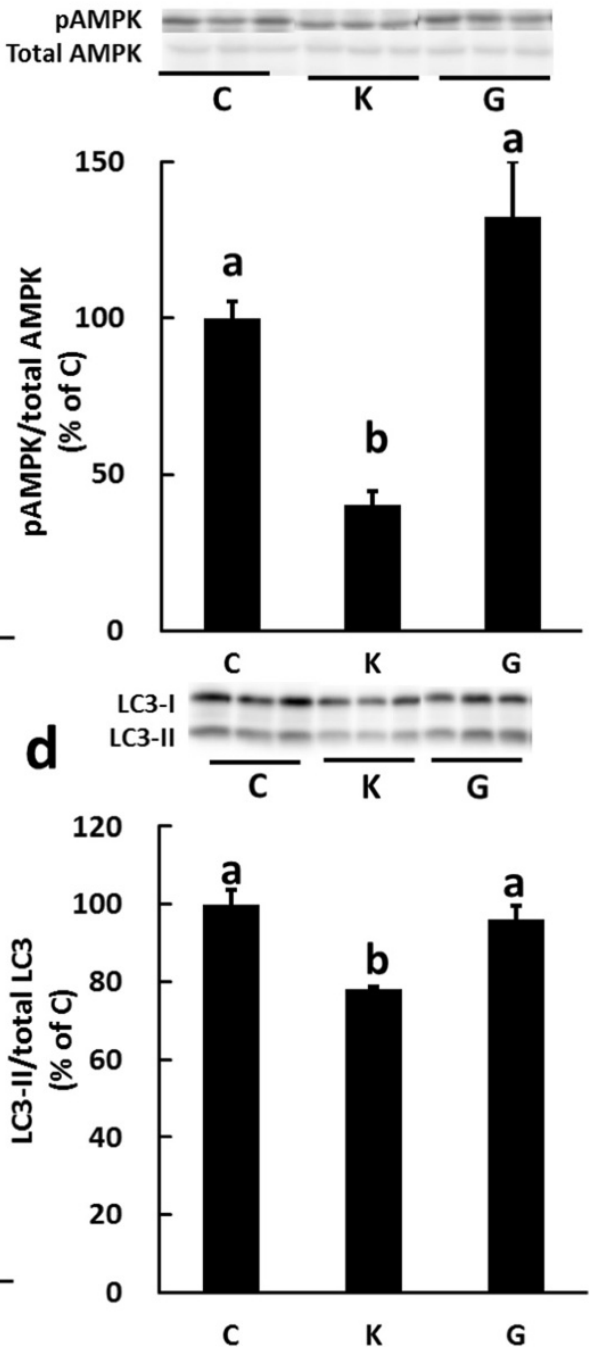

Figure 8 Comparison of Lys and Gly stimulation on the phosphorylation of Akt and AMPK, and on the activity of the autophagic-lysosomal system in C2C12 myotubes. Cells were untreated (C) or treated with $10 \mathrm{mM}$ Lys (K) or $10 \mathrm{mM}$ Gly (G) for 30 min. The phosphorylation levels of Ser473 in Akt (a), Thr308 in Akt (b), AMPK (c), and the ratio of LC3-II to total LC3 (LC3-I + LC3-II) (d), were determined in the lysates by Western blotting. Results are expressed as the level relative to that of the control group. Values are means with SE $(n=3-4)$. Different letters indicate significant differences among the groups $(p<0.05)$.

AICAR stimulation. When cells were treated with Lys in the presence of AICAR, the ratio of LC3-II to total LC3 remained suppressed, and Lys did not affect the activation of AMPK by AICAR (Figure $5 \mathrm{a}$ and $\mathrm{d}$ ). These results suggested that autophagic-lysosomal activity might be dominantly regulated by Akt rather than AMPK pathway in this study. Furthermore, the rate of myofibrillar protein degradation significantly increased in C2C12 cells treated with Lys and AICAR (AcK) compared to cells treated with Lys alone (K) (Figure 6). To investigate why myofibrillar protein degradation increased upon treatment with Lys and AICAR, we assessed the activity of the major proteolytic system, the ubiquitin-proteasomal system. The mRNA levels of MuRF1 and atrogin-1 were significantly increased in $\mathrm{C} 2 \mathrm{C} 12$ cells $30 \mathrm{~min}$ and $4 \mathrm{~h}$ following the addition of Lys and AICAR (AcK) compared to the other groups (C, Ac, K) (Figure 7). Therefore, the activation of AMPK in the presence of some amino acids may stimulate the ubiquitin-proteasomal system and increase the rate of myofibrillar protein degradation in $\mathrm{C} 2 \mathrm{C} 12$ cells, although the mechanism of this phenomenon is unclear.

On the other hand, AMPK has been shown to regulate protein synthesis through the mTOR pathway in $\mathrm{C} 2 \mathrm{C} 12$ 
cells (Williamson et al. 2006) and in rats (Bolster et al. 2002). When AMPK was phosphorylated by AICAR, mTOR signaling activity was significantly suppressed compared to in the presence of Lys alone (Figure $5 \mathrm{c}$ ). This result indicates that the suppressive effect of Lys on AMPK phosphorylation may help up-regulate protein synthesis by activating the mTOR pathway, rather than by regulating autophagic activity.

The nutritional value of Lys has been well-appreciated because Lys is a major limiting essential amino acid in some plant proteins, such as wheat gluten. Addition of Lys to low-Lys diet has been reported to help animal growth whereas animals which fed a low-Lys diet grow more slowly than those fed a diet containing adequate amounts of Lys (Nagao et al. 2010; Ishida et al. 2011). However, there is little information of molecular mechanisms in the health-promoting benefits of Lys until now. In this study, we indicated that Lys may act as regulator of autophagy and some signal molecules which control protein turnover in $\mathrm{C} 2 \mathrm{C} 12$ cells. The current results may provide basis for the development of nutritional treatment for muscle wasting and muscle mass gain by daily diet, and may contribute to the understanding the physiological function of amino acids and its molecular mechanism in skeletal muscle cells.

In conclusion, this study demonstrated for the first time that Lys suppresses myofibrillar protein degradation by regulating the autophagic-lysosomal system through phosphorylation of Akt. Consequently, Lys is an important regulatory amino acid in protein metabolism and exerts its effect via the Akt pathway.

\begin{abstract}
Abbreviations
Akt: Protein kinase B; Akti: Akt1/2 kinase inhibitor; AICAR: 5-aminoimidazole4-carboxamide-1- $\beta$-D-ribonucleoside; AMPK: Adenosine 5'-monophosphate (AMP)-activated protein kinase; BSA: Bovine serum albumin;

DMEM: Dulbecco's modified Eagle's medium; DMSO: Dimethyl sulfoxide; FBS: Fetal bovine serum; Gly: Glycine; HS: Horse serum; LC3: Light chain 3; Leu: Leucine; Lys: Lysine; MuRF1: Muscle ring-finger protein 1; mTOR: Mammalian target of rapamycin; PBS: Phosphate buffered saline; MeHis: 3-methylhistidine; 4E-BP1: elF4E-binding protein 1.
\end{abstract}

\section{Competing interests}

The authors declare that they have no competing interest.

\section{Authors' contributions}

TS performed the series of experiments and wrote the manuscript. YI and TN designed the study and wrote the manuscript. All authors read and approved the final manuscript.

\section{Author details}

${ }^{1}$ Department of Bioresources Science, The United Graduate School of Agricultural Sciences, Iwate University, Morioka, Iwate 020-8550, Japan. ${ }^{2}$ Department of Biological Chemistry and Food Science, Graduate School of Agriculture, Iwate University, Morioka, Iwate 020-8550, Japan.

Received: 3 September 2014 Accepted: 1 October 2014 Published: 8 October 2014

\section{References}

Arico S, Petiot A, Bauvy C, Dubbelhuis PF, Meijer AJ, Codogno P, Ogier-Denis E (2001) The tumor suppressor PTEN positively regulates macroautophagy by inhibiting the phosphatidylinositol 3-kinase/protein kinase B pathway. J Biol Chem 276:35243-35246

Bertrand L, Horman S, Beauloye C, Vanoverschelde JL (2008) Insulin signalling in the heart. Cardiovasc Res 79:238-248

Bolster DR, Crozier SJ, Kimball SR, Jefferson LS (2002) AMP-activated protein kinase suppresses protein synthesis in rat skeletal muscle through down-regulated mammalian target of rapamycin (mTOR) signaling. J Biol Chem 277:23977-23980

Coëffier M, Claeyssens S, Bensifi M, Lecleire S, Boukhettala N, Maurer B, Donnadieu N, Lavoinne A, Cailleux AF, Déchelotte P (2011) Influence of leucine on protein metabolism, phosphokinase expression, and cell proliferation in human duodenum. Am J Clin Nutr 93:1255-1262

Du M, Shen QW, Zhu MJ, Ford SP (2007) Leucine stimulates mammalian target of rapamycin signaling in C2C12 myoblasts in part through inhibition of adenosine monophosphate-activated protein kinase. J Anim Sci 85:919-927

Horman S, Vertommen D, Heath R, Neumann D, Mouton V, Woods A, Schlattner U, Wallimann T, Carling D, Hue L, Rider MH (2006) Insulin antagonizes ischemia-induced Thr172 phosphorylation of AMP-activated protein kinase alpha-subunits in heart via hierarchical phosphorylation of Ser485/491. J Biol Chem 281:5335-5340

Ishida A, Kyoya T, Nakashima K, Katsumata M (2011) Muscle protein metabolism during compensatory growth with changing dietary lysine levels from deficient to sufficient in growing rats. J Nutr Sci Vitaminol 57:401-408

Jamart C, Francaux M, Millet GY, Deldicque L, Frère D, Féasson L (2012) Modulation of autophagy and ubiquitin-proteasome pathways during ultra-endurance running. J Appl Physiol 112:1529-1537

Janku F, McConkey DJ, Hong DS, Kurzrock R (2011) Autophagy as a target for anticancer therapy. Nat Rev Clin Oncol 8:528-539

Kim JS, Wilson JM, Lee SR (2009) Dietary implications on mechanisms of sarcopenia: roles of protein, amino acids and antioxidants. J Nutr Biochem 21:1-13

Kimball SR, Shantz LM, Horetsky RL, Jefferson LS (1999) Leucine regulates translation of specific mRNAs in L6 myoblasts though mTOR-mediated changes in availability of elF4E and phosphorylation of ribosomal protein S6. J Biol Chem 274:11647-11652

Koopman R, van Loon $\sqcup$ (2009) Aging, exercise, and muscle protein metabolism. J Appl Physiol 106:2040-2048

Kovacic S, Soltys CL, Barr AJ, Shiojima I, Walsh K, Dyck JR (2003) Akt activity negatively regulates phosphorylation of AMP-activated protein kinase in the heart. J Biol Chem 278:39422-39427

Lang SM, Kazi AA, Hong-Brown L, Lang CH (2012) Delayed recovery of skeletal muscle mass following hindlimb immobilization in mTOR heterozygous mice. PLOS ONE 7:e38910

Lee MY, Jo SD, Lee JH, Han HJ (2008) L-leucine increases [ $\left.{ }^{3} \mathrm{H}\right]$-thymidine incorporation in chicken hepatocytes: involvement of the PKC, PI3K/Akt, ERK1/2, and mTOR signaling pathways. J Cell Biochem 105:1410-1419

Mammucari C, Milan G, Romanello V, Masiero E, Rudolf R, Del Piccolo P, Burden SJ, Di Lisi R, Sandri C, Zhao J, Goldberg AL, Schiaffino S, Sandri M (2008) FoxO3 controls autophagy in skeletal muscle in vivo. Cell Metab 6:458-471

Mascher H, Andersson H, Nilsson PA, Ekblom B, Blomstrand E (2007) Change in signaling pathways regulating protein synthesis in human muscle in the recovery period after endurance exercise. Acta Physiol 191:67-75

Mordier S, Deval C, Béchet D, Tassa A, Ferrara M (2000) Leucine limitation induces autophagy and activation of lysosome-dependent proteolysis in C2C12 myotubes through a mammalian target of rapamycin-independent signaling pathway. J Biol Chem 275:29900-29906

Nagao K, Bannai M, Seki S, Kawai N, Mori M, Takahashi M (2010) Voluntary wheel running is beneficial to the amino acid profile of lysine-deficient rats. Am J Physiol Endocrinol Metab 298:E1170-E1178

Nagasawa T, Kido T, Yoshizawa F, Ito Y, Nishizawa N (2002) Rapid suppression of protein degradation in skeletal muscle after oral feeding of leucine in rats. J Nutr Biochem 13:121-127

Naito T, Kuma A, Mizushima N (2013) Differential contribution of insulin and amino acids to the mTORC1-autophagy pathway in the liver and muscle. J Biol Chem 288:21074-21081

Nakashima K, Yakabe Y (2007) AMPK activation stimulates myofibrillar protein degradation and expression of atrophy-related ubiquitin ligases by increasing FOXO transcription factors in C2C12 myotubes. Biosci Biotechnol Biochem 71:1650-1656 

FoxO3a and interaction with Ulk1. J Cell Biochem 113:695-710

Sato T, Ito Y, Nagasawa T (2013) Regulation of skeletal muscle protein degradation and synthesis by oral administration of lysine in rats. J Nutr Sci Vitaminol (Tokyo) 59:412-419

Sato T, Ito Y, Nedachi T, Nagasawa T (2014) Lysine suppresses protein degradation through autophagic-lysosomal system in C2C12 myotubes. Mol Cell Biochem 391:37-46

Seldin MM, Lei X, Tan SY, Stanson KP, Wei Z, Wong GW (2013) Skeletal muscle-derived myonectin activates the mammalian target of rapamycin (mTOR) pathway to suppress autophagy in liver. J Biol Chem 288:36073-36082

Sugawara T, Ito Y, Nishizawa N, Nagasawa T (2007) Supplementation with dietary leucine to a protein-deficient diet suppresses myofibrillar protein degradation in rats. J Nutr Sci Vitaminol 53:552-555

Sugawara T, Ito Y, Nishizawa N, Nagasawa T (2009) Regulation of muscle protein degradation, not synthesis, by dietary leucine in rats fed a protein-deficient diet. Amino Acids 37:609-616

Suzuki T, Bridges D, Nakada D, Skiniotis G, Morrison SJ, Lin JD, Saltiel AR, Inoki K (2013) Inhibition of AMPK catabolic action by GSK3. Mol Cell 50:407-419

Tato I, Bartrons R, Ventura F, Rosa JL (2011) Amino acids activate mammalian target of rapamycin complex 2 (mTORC2) via PI3K/Akt signaling. J Biol Chem 286:6128-6142

Tisdale MJ (2009) Mechanisms of cancer cachexia. Physiol Rev 89:381-410

Vary TC, Lynch CJ (2004) Biochemical approaches for nutritional support of skeletal muscle protein metabolism during sepsis. Nutr Res Rev 17:77-88

Welle S (1999) Human Protein Metabolism. Springer-Verlag, New York, pp 196-227

Wolfe RR (2006) The underappreciated role of muscle in health and disease. Am J Clin Nutr 84:475-482

Williamson DL, Bolster DR, Kimball SR, Jefferson LS (2006) Time course changes in signaling pathways and protein synthesis in C2C12 myotubes following AMPK activation by AICAR. Am J Physiol Endocrinol Metab 291:E80-E89

Wilson GJ, Layman DK, Moulton CJ, Norton LE, Anthony TG, Proud CG, Rupassara SI, Garlick PJ (2011) Leucine or carbohydrate supplementation reduces AMPK and eEF2 phosphorylation and extends postprandial muscle protein synthesis in rats. Am J Physiol Endocrinol Metab 301:E1236-E1242

Young VR, Munro HN (1978) N'-methylhistidine (3-methylhistidine) and muscle protein turnover: an overview. Fed Proc 37:2291-3000

Zeanandin G, Balage M, Schneider SM, Dupont J, Hébuterne X, Mothe-Satney I, Dardevet D (2012) Differential effect of long-term leucine supplementation on skeletal muscle and adipose tissue in old rats: an insulin signaling pathway approach. Age (Dordr) 34:371-387

Zhao J, Brault JJ, Schild A, Cao P, Sandri M, Schiaffino S, Lecker SH, Goldberg AL (2007) FoxO3 coordinately activates protein degradation by the autophagic/ lysosomal and proteasomal pathways in atrophying muscle cells. Cell Metab 6:472-483

doi:10.1186/2193-1801-3-584

Cite this article as: Sato et al:: Lysine suppresses myofibrillar protein degradation by regulating the autophagic-lysosomal system through phosphorylation of Akt in C2C12 cells. SpringerPlus 2014 3:584.

\section{Submit your manuscript to a SpringerOpen ${ }^{\circ}$ journal and benefit from:}

- Convenient online submission

- Rigorous peer review

- Immediate publication on acceptance

- Open access: articles freely available online

- High visibility within the field

- Retaining the copyright to your article

Submit your next manuscript at $\gg$ springeropen.com 